AB0073

INTESTINAL PERMEABILITY IN SPONDYLOARTHRITIS: A SYSTEMATIC REVIEW OF THE LITERATURE

S. Hecquet ${ }^{1}$, P. Totoson ${ }^{2}$, H. Martin ${ }^{2}$, C. Prati ${ }^{1}$, D. Wendling ${ }^{1}$, C. Demougeot ${ }^{2}$, F. Verhoeven ${ }^{1}{ }^{1}$ Centre hospitalier régional universitaire de Besançon, Rheumatology, Besançon, France; ${ }^{2}$ EA4267 PEPITE LAB, Physiology, Besançon, France

Background: Growing evidence argue for a role of the gut in the pathophysiology of various chronic rheumatic diseases such as spondyloarthritis $(\mathrm{SpA})$. This so-called "gut-joint axis" involves dysbiosis, bacterial translocation, intestinal inflammation and increase in intestinal permeability. Recent data from clinical and basic research suggested that the integrity of the intestinal barrier might be a key determinant in translating autoimmunity to inflammation, making intestinal permeability a potential marker or a target for future therapies.

Objectives: To analyse the available data on intestinal permeability in SpA patients and the effects of drugs such as non-steroidal anti-inflammatory drugs (NSAIDs) on intestinal permeability.

Methods: A systematic review was conducted. Without date restriction, the following databases were searched through September 1, 2020: Medline, Embase and Cochrane. Studies with patients with SpA assessing the intestinal permeability were selected. Some of the included studies have assessed the effect of NSAIDs on intestinal permeability.

Results: A total of 12 studies were included in the final analysis. The 12 studies involved a total of $268 \mathrm{SpA}$ patients, including 240 ankylosing spondylitis (AS). Among the studies included, four studies used the lactulose/mannitol test, four studies used the 51Cr-ethylenediaminetetraacetic test and two studies used the polyethylene glycols test. Nine of the 12 studies reported increased intestinal permeability regardless on the method used for intestinal permeability evaluation. Four studies evaluated the link between disease activity, assessed by CRP and ESR levels, and intestinal permeability and showed no correlation between increased intestinal permeability and markers of disease activity in AS patients. As regards the effects of NSAIDs on intestinal permeability, data are controversial. Two studies, including one evaluating indomethacin, did not show any influence of NSAIDs in AS patients, one study showed an increase in intestinal permeability under NSAIDs in only $60 \%$ of the patients, another study reported increased intestinal permeability. When comparing the effect of NSAIDs in patients with AS to healthy subjects, one study reported a comparable NSAIDs-induced increase in intestinal permeability in both groups. Conclusion: The results of our review suggest that increased intestinal permeability is present in SpA patients even in the absence of NSAIDs use and regardless of the method used to assess intestinal permeability. The effects of NSAIDs on intestinal permeability in SpA patients is more controversial and further studies are needed to clarify them.

Disclosure of Interests: None declared

DOI: 10.1136/annrheumdis-2021-eular.3254

\section{AB0074 \\ NO DIFFERENCE BETWEEN THE SEROLOGIES OF DENTAL GERMS AND THE PHENOTYPES OF SPONDYLOARTHRITIS WITHIN THE DESIR COHORT}

T. Neel ${ }^{1}$, A. Tournadre ${ }^{2}$, M. Paul ${ }^{3}$, M. Norman ${ }^{3}$, S. Paul ${ }^{4}$, H. Marotte ${ }^{1} .{ }^{1} \mathrm{CHU}$, Rhumatology, Saint-Etienne, France; ${ }^{2} \mathrm{CHU}$, Rhumatology, Clermont-Ferrand, France; ${ }^{3}$ SAINBIOSE INSERM U1059, Research, Saint-Etienne, France; ${ }^{4}$ GIMAP EA3064, Immunology, Saint-Etienne, France

Background: Porphyromonas gingivalis (PG) and Prevotella Intermedia $(\mathrm{PI})$ are two oral pathogens involved in the pathophysiology of chronic periodontopathies. Several studies have determined a role of these periodontal pathologies in the pathophysiology of rheumatoid arthritis (1) while the link with spondyloarthritis is not clearly established with currently contradictory studies $(2,3,4)$.

Objectives: The purpose of this work is to investigate a link between spondylarthritis and the presence of chronic periodontopathy evaluated by PG and IP serologies.

Methods: The positivity and quantity of anti-PG and anti-PI antibodies were determined by ELISA method in patients from the DESIR cohort with one of the spondyloarthritis phenotypes compared to patients with common low back pain $(n=50)$ (population control). Patients with spondyloarthritis were classified according to the following phenotypes (diagnosis chosen at 3 years in the DESIR cohort): axial spondylarthritis $(n=126)$, psoriatic rheumatism $(n=101)$, spondyloarthritis associated with chronic inflammatory bowel disease $(n=36)$, undifferentiated spondylitis $(n=241)$, reactive arthritis $(n=2)$, SAPHO (acronym for Synovite, Acne, Pustulose, Hyperostosis and Osteitis. Correlations between patients' serological status, and smoking status, body mass index and age were sought.

Results: According to the general characteristics, the control group was significantly older than the axial spondylarthritis $(p<0.001)$, and the psoriatic rheumatism group had a higher body mass index than the control population $(p<0.05)$. The positivity and concentration of anti-PG and anti-PI antibodies were similar between different groups of spondyloarthritis compared to the control group. However, the subgroups "reactive arthritis" and "SAPHO" could not be analyzed due to insufficient staffing. Smoking status and body mass index were not related to antibody concentrations, however there was a statistical correlation between anti-PG antibody concentrations and age. After adjusting on age, there was no difference between the axial spondylarthritis and control groups.

Conclusion: Our results therefore suggest the absence of a link between periodontal germs involved in chronic periodontopathies and spondyloarthritis, provided that the two phenotypes involving germs in their pathophysiology could not be analyzed. Our results from a population of beginner spondylitis are therefore not in favour of the involvement of oral flora in the pathophysiology of spondyloarthritis, as is the case in rheumatoid arthritis (1). The associations found previously could therefore be favoured by a systemic inflammatory phenomenon. In conclusion, our study suggests no link between chronic periodontopathy and the occurrence of spondyloarthritis. However, the effect of chronic periodontopathy on the evolution of spondylarthritis remains to be explored.

\section{REFERENCES:}

[1] Detert J, Pischon N, Burmester GR, Buttgereit F. The association between rheumatoid arthritis and periodontal disease. Arthritis Res Ther 2010;12(5):218.

[2] Ratz T, Dean LE, Atzeni F, Reeks C, Macfarlane GJ, Macfarlane TV. A possible link between ankylosing spondylitis and periodontitis: a systematic review and meta-analysis. Rheumatology. 2015;54(3):500-10.

[3] Pischon N, Pischon T, Gülmez E, Kröger J, Purucker P, Kleber B-M, et al. Periodontal disease in patients with ankylosing spondylitis. Ann Rheum Dis. janv 2010;69(01):34-8.

[4] Sezer U, Erciyas K, Pehlivan Y, Üstün K, Tarakçıŏlu M, Şenyurt SZ, et al. Serum cytokine levels and periodontal parameters in ankylosing spondylitis: Ankylosing spondylitis and periodontal diseases. J Periodontal Res. juin 2012;47(3):396-401.

Disclosure of Interests: None declared

DOI: 10.1136/annrheumdis-2021-eular.3258

\section{AB0075 HSA_CIRC_0012732, HSA_CIRC_0008961, HSA_CIRC_0405239 AND HSA_CIRC_0068784 MIGHT BE INVOLVED IN THE PATHOGENESIS AND DEVELOPMENT OF ANKYLOSING SPONDYLITIS}

Y. P. Tang ${ }^{1}$, Y. F. Qing ${ }^{1}$, Z. R. Dong ${ }^{1}$, F. Dai ${ }^{1}$, J. Zheng ${ }^{1}$, Y. Jiang ${ }^{1}$, Y. X. He ${ }^{1}$, Q. B. Zhang ${ }^{2}{ }^{1}$ Institute of Rheumatology and Immunology, Affiliated Hospital of North Sichuan Medical College, Department of Rheumatology and Immunology, Affiliated Hospital of North Sichuan Medical College, Nanchong, China; ${ }^{2}$ Institute of Rheumatology and Immunology, Affiliated Hospital of North Sichuan Medical College, Department of Geriatrics, Affiliated Hospital of North Sichuan Medical College, Nanchong, China

Background: CircRNAs have been found to be involved in the occurrence and development of many rheumatic diseases[1-2]. Are circRNAs involved in the pathogenesis of ankylosing spondylitis (AS)? How do these circRNAs participate in the pathogenesis of AS? This all needs further study.

Objectives: This study is to clarify the expression levels of hsa_circ_0012732, hsa_circ_0008961, hsa_circ_0405239 and hsa_circ_0068784 in the peripheral blood of AS patients, and to explore whether these circRNAs are involved in the pathogenesis of AS.

Methods: To collected 60 cases of AS (30 cases of active AS (ASA): BASDA> 6 or $6>$ BASDAl $>4$, ESR $>22 \mathrm{~mm} / \mathrm{h}$ or $6>$ BASDAl $>4$, hsCRP $>9 \mathrm{mg} / \mathrm{L} ; 30$ cases of stable AS (ASS): BASDAI <4) and 30 health control $(\mathrm{HC})$ peripheral blood samples, related clinical and laboratory indicators. The relative expression levels of hsa circ 0012732, hsa circ 0008961, hsa circ 0405239 and hsa circ_0068784 in each group were detected by real-time quantitative polymerase chain reaction (qPCR). The relationships between the 4 circRNAs and clinical and laboratory indicators were explored by correlation analysis.

Results: 1. The qPCR results suggested that the expression of hsa circ 0012732 between the ASA and ASS groups was statistically significant $(p<0.05)$, and the expression of hsa_circ_0008961 was statistically significant between the ASA and HC groups $(p<0.05)$. Howeverthere was no statistical significance among other groups $(p>0.05)$ Figure 1. Similarly, the expression level of hsa circ_0405239 was not statistically significant among the groups ( $p>0.05)$, and the same was true for hsa_circ_0068784 ( $p>0.05)$.

2. Correlation analysis results (Figure 2) showed that hsa circ 0012732 is positively correlated with lymphocyte count (LY), mean corpusular volume (MCV), albumin (ALB), and negatively correlated with Bath ankylosing spondylitis disease activity index (BASDAI), Bath ankylosing spondylitis functional index (BASFI), high sensitivity C-reactive protein (hsCRP), Globulin (GLOB) $(p<0.05)$; hsa_circ_0008961 is negatively correlated with platelet $(P L T)(p<0.05)$ hsa_circ_0405239 is negatively correlated with BASDAI and BASFI; hsa circ 0068784 was negatively correlated with BASDAI $(p<0.05)$; and there was no statistically significant $(p>0.05)$ between these 4 circRNAs and other indicators. 
Conclusion: Hsa_circ_0012732, hsa_circ_0008961, hsa_circ_0405239 and hsa_circ_0068784 may be related to the pathogenesis of AS. Among them, hsa_circ_0012732 may be involved in AS inflammation and has the potential to participate in the judgment of disease activity.

REFERENCES:

[1] LS, K., et al., The biogenesis, biology and characterization of circular RNAs. 2019. 20(11): p. 675-691.

[2] J, W., et al., Non-coding RNAs in Rheumatoid Arthritis: From Bench to Bedside. 2019. 10: p. 3129

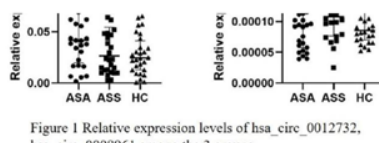

Figure 1 Relative expression levels of hsa_circ_0012732.
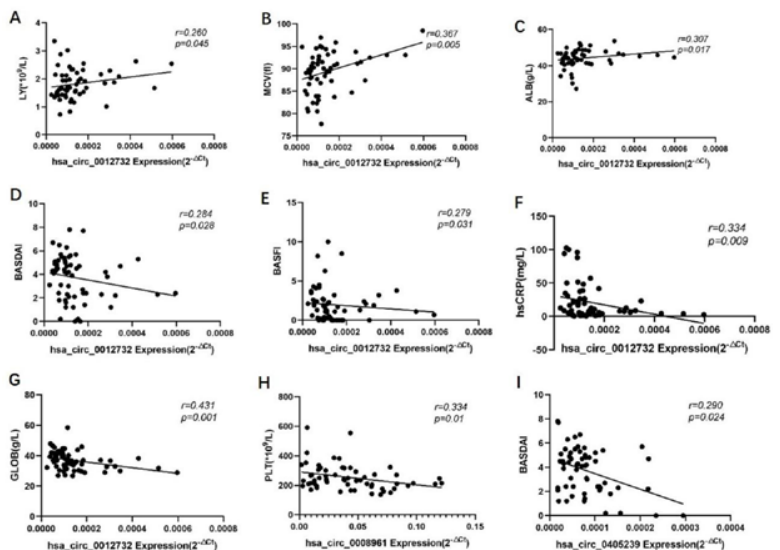

${ }^{3}{ }_{15}^{10}$.

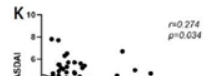

Disclosure of Interests: Yi-Ping Tang Grant/research support from: Science and Technology Project of Nanchong City (18SXHZ0522)., Yu-Feng Qing: None declared, Zeng-Rong Dong: None declared, Fei Dai: None declared, Jianxiong Zheng: None declared, Yi Jiang: None declared, Yi-Xi He: None declared, Quan-Bo Zhang Grant/research support from: National Natural Science Foundation of China(General Program) (81974250); Science and Technology Plan Project of Sichuan Province (2018JY0257)

DOI: 10.1136/annrheumdis-2021-eular.3412

\section{$\mathrm{AB} 0076$ \\ THE ROLE OF MACROPHAGE MIGRATION INHIBITORY FACTOR IN ACTIVITY AND PROGRESSION OF ANKYLOSING SPONDYLITIS}

Y. Gazar ${ }^{1}$, M. Hanafy ${ }^{1}$, M. Elrefi ${ }^{2} .{ }^{1}$ Faculty of Medicine, AL-Azhar university, Rheumatology and Rehabilitation, cairo, Egypt; ${ }^{2}$ Military Hospitals, Rheumatology, cairo, Egypt

Background: Ankylosing Spondylitis (AS) is a chronic inflammatory form of arthritis that predominantly affects the axial joints and has a global prevalence of $1 \%$. Disease progression is marked by osteoproliferation and bone fusion in the axial joints, causing considerable loss of mobility. Migration Inhibitory Factor (MIF) is a potent pro-inflammatory cytokine implicated in several diseases. Elevated serum MIF levels have been reported in AS the role of MIF in bone formation has been a subject of controversy.

Objectives: Detection the level of Migration Inhibitory Factor in Ankylosing Spondylitis patients and correlate the level with disease activity and progression. Methods: This cross-sectional case-control study were concluded upon 70 randomly selected people from internal medicine department in Kobry El-kobba Military Hospital and from rheumatology and rehabilitation department in EL-Hussein university hospital. The population study were divided into two groups: Group A: (50) Patients with Ankylosing Spondylitis (AS) who met the European Spondyloarthropathy Study Group (ESSG) criteria for AS (30) and Group B: (20) Healthy controls. Serum MIF by ELISA were measured. X-rays cervical \&lumbosacral vertebrae (dorsal, lateral) views were obtained. m-SASS score (based on radiographic findings to detect progression) were assessed. MSK U/S on Tendo Achilles affected in AS patients were done.

Results: the mean age of all patients was $(38.2 \pm 8.4)$ years. Regarding gender of the patients, the majority $(65.7 \%)$ of patients were males, while $(34.3 \%)$ were females. $(88 \%)$ of AS patients had cervical erosion or sclerosis, $(46 \%)$ had dorsal erosion or sclerosis, (92\%) had lumbosacral erosion or sclerosis, with m-SASS score of $(11 \pm 6.1)$. Regarding musculoskeletal U/S, $(72 \%)$ had evidence of inflammation and erosion. Highly significant increase in ESR, CRP and MIF levels in A group; compared to control B group; with highly significant statistical difference $(p<0.01)$. the increase in smoking, ESR and MIF level; had an independent effect on increasing the probability of progression occurrence; with significant statistical difference ( $p<0.05$ respectively). MIF level at a cutoff point $(>51)$ predicted patients with progression, with fair accuracy $(74 \%)$, sensitivity= $53 \%$ and specificity $=94 \%(p=0.0056)$.

Conclusion: MIF ply an important role in the pathogenesis of Ankylosing Spondylitis and has a link between chronic inflammation, new bone formation and disease progression and can be used as indicator for activity and disease progression.

Disclosure of Interests: None declared

DOI: 10.1136/annrheumdis-2021-eular.3421

\section{SLE, Sjögren's and APS - aetiology, pathogenesis and animal models}

\section{AB0077 ASSOCIATION BETWEEN VITAMIN D RECEPTOR GENE POLYMORPHISMS AND SYSTEMIC LUPUS ERYTHEMATOSUS IN MALTESE PATIENTS}

R. Magro ${ }^{1}$, J. A. Grech Meli ${ }^{2}$, J. Debattista3, N. Aquilina ${ }^{4}$, K. Gatt5, A. Borg6,

C. Scerri ${ }^{2}{ }^{1}$ Mater Dei Hospital, Rheumatology, Msida, Malta; ${ }^{2}$ University of Malta, Faculty of Medicine and Surgery, Msida, Malta; ${ }^{2}$ University of Malta, Faculty of Medicine and Surgery, Msida, Malta; ${ }^{4}$ Mater Dei Hospital, Medicine, Msida, Malta; ${ }^{4}$ Mater Dei Hospital, Medicine, Msida, Malta; ${ }^{1}$ Mater Dei Hospital, Rheumatology, Msida, Malta

Background: Vitamin D deficiency is highly prevalent in patients with systemic lupus erythematosus (SLE). Vitamin D acts through the vitamin D receptor (VDR) that is present in most cells, and it can regulate the transcription of over 200 genes. The expression of vitamin $D$ receptors by a variety of cells belonging to the innate and adaptive immune systems has created interest with regards to the role of vitamin $D$ in the pathogenesis of SLE. Several polymorphisms of the VDR gene have been described, namely Bsml, Apal, Taql and Fokl. A number of VDR gene polymorphism genotypes have been associated with increased risk of SLE mostly in Asians and Africans. ${ }^{1}$

Objectives: The aim of this study was to establish whether an association was present between VDR gene polymorphisms and SLE susceptibility in a cohort of SLE patients living in Malta. A further aim was to assess the relationship between these VDR gene polymorphisms and SLE disease characteristics.

Methods: 59 SLE patients living in Malta and attending Rheumatology clinic at Mater Dei Hospital were recruited for the study after providing informed consent. The patients were over the age of 18 years and fulfilled the SLICC classification criteria for SLE. The patients were interviewed and blood samples were taken. RNA extraction was performed from whole blood. QuantiGene Plex technology was used to measure the expression of 12 interferon (IFN) signature genes in the extracted RNA. 93 cord blood samples obtained from individuals living in Malta were used as a control. DNA extraction was carried out from the blood samples obtained from the patients and controls. The VDR gene was screened and the regions containing the VDR polymorphisms were amplified for each patient. The amplified regions were then digested with their respective restriction enzymes in order to view the patient's genotype via restriction fragment length polymorphism. Statistical analysis, including odds ratio (OR), was carried out to gauge the significance in the association of these polymorphisms with SLE.

Results: $94.9 \%$ of SLE patients were female and they had a mean age of 44.5 years. All the patients were of Caucasian ethnicity. $13.6 \%$ had vitamin $D$ deficiency (serum 25-hydroxyvitamin D $<20 \mathrm{ng} / \mathrm{ml}$ ) and $25.4 \%$ were vitamin $\mathrm{D}$ insufficient (serum 25-hydroxyvitamin D 20-29ng/ml). The results showed that when Apal polymorphism was present as a homozygote for the variant allele there was a significant decrease in SLE prevalence $(\mathrm{OR}=0.39, \mathrm{Cl} 0.17-0.87, \mathrm{p}=0.02)$. The results were also analysed by placing the polymorphs into haplotypes. The haplotype containing all wild-type alleles for the VDR gene and the haplotype containing al wild-type alleles with the variant allele for Fokl had an increased prevalence of SLE ( $O R=1.95, \mathrm{Cl} 1.12-3.38, \mathrm{p}=0.01$ and $\mathrm{OR}=2.36, \mathrm{Cl}$ 1.13-4.91, $\mathrm{p}=0.02$ respectively). The patients who were homozygous for the variant allele for Bsml had a significantly higher SLE disease activity index-2K (SLEDAI-2K) (mean 5.00) compared to those that were heterozygous (mean 2.66; $p=0.010$ ). No significant difference was noted in damage, IFN signature gene expression, organ manifestation and autoantibody profile between the different genotypes for the 4 VDR polymorphisms. SLE patients who were homozygous variant for the Apal or Taql polymorphisms had an increased prevalence of fibromyalgia $(\mathrm{OR}=7.50, \mathrm{Cl} 1.47-$ 38.16, $\mathrm{p}=0.02$ and $\mathrm{OR}=12.00, \mathrm{Cl} 1.80-80.05, \mathrm{p}=0.02$ respectively).

Conclusion: The study showed that in the Maltese population the presence of the VDR gene polymorphism haplotype containing all wild-type alleles and the haplotype containing all wild-type alleles with the variant allele for Fokl are associated with an increased risk of SLE. Moreover the homozygous variant genotype for Bsml was associated with a higher SLE disease activity. The homozygous 